\title{
Distinct eGFR trajectories are associated with risk of myocardial infarction in people with diabetes or pre-diabetes
}

\section{Yingting Zuo}

Capital Medical University

\section{Anxin Wang}

Beijing Tiantan Hospital

\section{Shuohua Chen}

Kailuan General Hospital

\section{Xue Tian}

Capital Medical University

Shouling Wu ( $\nabla$ drwusl@163.com)

Kailuan General Hospital https://orcid.org/0000-0001-7095-6022

\section{Yan He}

Capital Medical University

\section{Original investigation}

Keywords: estimated glomerular filtration rate (eGFR); trajectory; myocardial infarction; cohort study

Posted Date: May 5th, 2020

DOl: https://doi.org/10.21203/rs.3.rs-25392/v1

License: (1) This work is licensed under a Creative Commons Attribution 4.0 International License. Read Full License 


\section{Abstract \\ Background}

The relationship between estimated glomerular filtration rate (eGFR) trajectories and myocardial infarction (MI) remains unclear in people with diabetes or prediabetes. We aimed to identify common eGFR trajectories in people with diabetes or prediabetes and to examine their association with MI risk.

\section{Methods}

The data of this analysis was derived from the Kailuan study, which was a prospective community-based cohort study. The eGFR trajectories of 24,723 participants from year 2006 to 2012 were generated by latent mixture modeling. Incident cases of Ml occurred during 2012 to 2017, confirmed by review of medical records. Cox proportional hazards models were used to calculate hazard ratios (HR) and their $95 \%$ confidence intervals (Cls) for the subsequent risk of MI of different eGFR trajectories.

\section{Results}

We identified 5 distinct eGFR trajectories, and named them as low-stable (9.4\%), moderate-stable (31.4\%), moderate-increasing (29.5\%), high-decreasing (13.9\%) and high-stable (15.8\%) according to their range and pattern. During a mean follow-up of 4.61 years, there were a total of 235 incident MI. Although, the high-decreasing group had similar eGFR levels with the moderate-stable group at last exposure period, the risk was much higher (adjusted HR, 3.43; $95 \% \mathrm{Cl}, 1.56-7.54$ versus adjusted $\mathrm{HR}, 2.82 ; 95 \% \mathrm{Cl}, 1.34-5.95$ ). Notably, the moderate-increasing group had reached to the normal range, still had a significantly increased risk (adjusted $\mathrm{HR}, 2.55 ; 95 \% \mathrm{Cl}, 1.21-5.39$ ).

\section{Conclusions}

eGFR trajectories were associated with MI risk in people with diabetes or prediabetes. Emphasis should be placed on early and long-term detection and control of eGFR decreases to further reduce MI risk.

\section{Background}

Chronic kidney disease (CKD) is affecting $10-16 \%$ of the global adult population[1], and was the significant cause of several adverse clinical outcomes such as kidney failure[2] ,cardiovascular disease[3] and all-cause mortality[4]. Estimated glomerular filtration rate (eGFR) is a common and convenient indicator of CKD. Associations with myocardial infarction (MI) were less consistent, with some studies showing a significantly increased risk[5-7], and others observing an unchanged risk with lower estimated eGFR $[8,9]$. Since these studies measured eGFR at only one single time point, there has been no 
consideration of how eGFR varies within individuals over time and its potential impact on the future risk, which may not be enough for characterizing the long-term Ml risk prediction.

Recently, changes in eGFR was measured with two major methods: percentage change using two measurements and slope-based approaches using multiple measurements[10-12], both of which may largely ignore trajectory of eGFR over time. Accordingly, the African American Study of Kidney Disease and Hypertension (AASK) have used eGFR trajectory to reflect the change of eGFR in long-term exposure[13], further to determine the associations between annual change in eGFR and subsequent clinical outcomes. Although, some currently emerging reports pay attention to the eGFR trajectories, only focus on the risk of all-cause mortality[14, 15].

In addition, a meta-analysis suggested that insulin resistance might aggravate the decrease in eGFR, leading to renal impairment in participants with diabetes or prediabetes[16]. Simultaneously, diabetes or prediabetes was associated with increased MI risk. Thus, it is important to estimate the association between eGFR trajectories and risk of MI in the diabetes and prediabetes population.

Therefore, in present study, we aimed to identify distinct trajectories of eGFR in the diabetes or prediabetes population, have a better clarification of the association between eGFR and the risk of MI and thereby to provide effective prevention strategies for MI.

\section{Methods}

\section{Study Population}

This study consisted of 101,510 participants ( 81,110 males and 20,400 females, $18-98$ years old) at baseline (2006) in the Kailuan Study is a community-based longitudinal cohort study which is located in the Tangshan city, China[17]. The study design, methods, rationale and examination details of the study have been previously described[8]. All participants provided their written informed consent and finished questionnaire interviews, anthropometric measurements, clinical examinations, and laboratory assessments in 2-yearcycles until the present day. This investigation was approved jointly by the Ethics Committee of the Kailuan General Hospital and Beijing Tiantan Hospital. In the present study, we included participants, which with impaired glucose metabolism at 2006, had at least 2 measurements of eGFR levels during the exposure period (from 2006 to 2012) (Figure 1) and free of MI in or prior to 2012, and (Figure 2).

\section{Assessment of eGFR}

Serum creatinine (Scr) sample was collected in the morning after an overnight fast and was analyzed using an automatic biochemical analyzer (Hitachi 747, Tokyo, Japan). eGFR was calculated using the creatinine-based Chronic Kidney Disease Epidemiological Collaboration (CKD-EPI) equation[18] and at least 2 measurements during the exposure period (from 2006 to 2012) were used to establish the eGFR trajectory. 


\section{Assessment of Outcome}

After the baseline examination, participants were followed for the first occurrence of MI. The incidence of MI was identified by referring to medical records from all 11 Kailuan hospitals and the Municipal Social Insurance Institution from the baseline examination to December 31, 2017. The diagnostic criteria of MI were based on chest pain symptoms, electrocardiogram (ECG) changes, and cardiac enzyme levels, collectively[19].

\section{Assessment of Covariates}

Demographic and clinical characteristics, including age, gender, smoking status, alcohol status, physical activity, history of disease and medical history were obtained using a via questionnaires. Smoking and alcohol status were classified as never, former or current according to self-reported information. The physical activity was classified as inactive (none), moderate active ( $<80$ per week) and very active ( $\geq 80$ minutes of activity per week). Dyslipidemia was defined as any self-reported history. Hypertension was defined as any self-reported hypertension or BP $\geq 140 / 90 \mathrm{~mm} \mathrm{Hg}$. Diabetes mellitus was defined as any self-reported diabetes mellitus or fasting blood glucose $(\mathrm{FBG}) \geq 7 \mathrm{mmol} / \mathrm{L}$. Anthropometric measurements were measured by trained doctors and nurses, including weight, height, and blood pressure (BP). Body mass index (BMI) was calculated as weight $(\mathrm{kg}) /$ height $(\mathrm{m})^{2}$. Systolic blood pressure (SBP) and diastolic blood pressure (DBP) were measured 3 times with the participants in the seated position using a mercury sphygmomanometer, and the average of 3 readings was used in the analyses.

Blood sample was collected in the morning after an overnight fast and was analyzed using an autoanalyzer (Hitachi 747, Tokyo, Japan). Fasting blood glucose (FBG), lipids profile including total cholesterol (TC), triglycerides (TG), low-density lipoprotein cholesterol (LDL-C), and high-density lipoprotein cholesterol (HDL-C), were measured using a standardized method. Using a color scale, the results of the urine strip test were semi quantified as absent, trace, $1+, 2+$ or $3+$ and proteinuria was defined as 1+ or higher. All the above items were measured or updated 2-yearcycles (from 2006 to 2012).

\section{Statistical Analyses}

In current study, we used eGFR trajectory groups as the exposure. These models were identified by the latent mixture modeling (SAS PROC TRAJ)[20, 21]. The model with 5 patterns was identified to fit the best. We then qualitatively examined the trajectory groups and named each trajectory group based on their visual patterns of change in eGFR levels.

Continuous and categorical variables were described as median with inter-quartile range and percentages, and were used Wilcoxon and chi-square to test the significance of differences, respectively, between trajectory groups. Person-years were calculated from the data in 2012 to the date when MI occurred, the date of death or the date of participating in the last examination in this analysis. 
Cox proportional hazards models were used to investigate the association of trajectory groups with the risk of Ml. Cox proportional hazard models were built to adjust for different confounding factors: Model 1 was unadjusted. Model 2 was adjusted for age and gender at baseline. Model 3 was further adjusted for current smoker, current alcohol, physical activity, proteinuria, ACEI medication and BMI, FBG, SBP, TC at baseline. Because these potential confounders may change during the follow-up, we conducted several sensitivity analyses with adjustment all potential confounders in 2012 or average BMI, FBG, SBP and TC during the exposure period (from 2006 to 2012). Besides, we also performed adjusting survival curves relative hazards from the cox proportional hazards models[22].

It is unclear whether the eGFR trajectories in special population might exhibit differential risk of MI. We further explored potential interaction between eGFR trajectories and age ( $<55$ years versus[vs] $\geq 55$ years), gender, diabetes status (diabetes vs pre-diabetes), hypertension status (yes vs no) and proteinuria (yes vs no).

Statistical analyses were conducted by using SAS version 9.4 (SAS Institute, Cary, NC, USA). All reported $P$ values were based on 2-sided tests of significance, and $P<0.05$ was deemed statistically significant.

\section{Results}

\section{Characteristics of the Trajectory Groups}

In this prospective cohort of 24,723 participants with diabetes or prediabetes, we identified 5 eGFR distinct trajectories during a 6-year follow up exposure period. Each of the trajectory groups were named according to their range and visual patterns of change in eGFR levels: $9.4 \%$ of participants had the "lowstable" trajectory, in which they maintained low eGFR levels throughout the exposure period; $31.4 \%$ of participants had the "moderate-stable" trajectory, in which they maintained moderate eGFR levels throughout the exposure period; $29.5 \%$ of participants had the "moderate-increasing" trajectory, in which they started with moderate eGFR levels and then experienced a rapid increase in eGFR levels; $13.9 \%$ of participants had the "high-decreasing" trajectory, in which they started with relatively high eGFR levels and then experienced a decrease in eGFR levels; $15.8 \%$ of participants had the "high-stable" trajectory, in which they maintained high levels of eGFR during the exposure period (Figure 3 ).

Characteristics in baseline (2006) of the five trajectories were compared and are summarized in Table 1. Compared with the low-sable group, participants in the high-stable groups were more likely to be older and women, have higher BMI, less drinking, less smoking, and higher concentrations of TC, FBG, SBP and DBP (Table 1).

\section{eGFR Trajectories Associated with Incident MI}

There was a total of 235 incidents of MI during a mean follow-up of $4.61 \pm 0.82$ years. Table 2 shows that the association between eGFR trajectories and the risk of MI in diabetes or prediabetes participants. Figure 4 shows that the risk of MI increased significantly in accordance with the eGFR trajectory groups 
from high-stable to low-stable $(P<0.01)$. We observed that eGFR trajectories were significantly associated with future MI risk. After adjustment for potential confounders, compared with participants in the highstable group, those in the low-stable group (hazard ratio [HR], 4.54; 95\% confidence intervals (Cls), 2.0310.12) experienced the highest risk of $\mathrm{MI}$ among all 5 trajectory groups. Although participants in the moderate-stable group had eGFR levels similar to those in participants in the high-decreasing group at last exposure period $\left(76.3 \mathrm{ml} / \mathrm{min} / 1.73 \mathrm{~m}^{2} \mathrm{vs} 76.8 \mathrm{ml} / \mathrm{min} / 1.73 \mathrm{~m}^{2}\right)$, the risk of Ml was much higher in these participants with eGFR decreasing over time than in those with eGFR stable over time (HR, 2.82; $95 \% \mathrm{Cl}, 1.34-5.95$ vs $\mathrm{HR}, 3.43 ; 95 \% \mathrm{Cl}, 1.56-7.54)$. Notwithstanding, the change in eGFR levels increasing reach to the normal range, participants in the moderate-increasing group still had a significantly increased risk of $\mathrm{MI}(\mathrm{HR}, 2.55 ; 95 \% \mathrm{Cl}, 1.21-5.39)$ as compared with those in the high-stable group after adjustment potential confounders.

\section{Sensitivity Analysis}

Considering these potential confounders change from 2006 to 2012, we add sensitivity analyses by adjustment for covariates in 2012 or average BMI, FBG, SBP and TC during the exposure period (from 2006 to 2012), and the results remained the same (Table 2). Taken together, the results of our study indicated that the trajectories of eGFR were important for the development of MI.

\section{Subgroup Analysis}

The associations between eGFR trajectories and incident MI stratified by age, gender, hypertension, diabetes and proteinuria are shown in Table 3. We also tested the interactions between eGFR trajectories and age, gender, hypertension, diabetes and proteinuria in relation to $\mathrm{Ml}$ and subtypes. We did not find significant interactions between eGFR trajectories and age, gender, hypertension, diabetes and proteinuria in relation to both subtypes of $\mathrm{Ml}$ ( $P$ for interaction $>0.05$ for all).

\section{Discussion}

In this large prospective cohort of people with diabetes and pre-diabetes, we identified 5 distinct eGFR trajectory groups, in which participants shared a similar pattern of change in eGFR levels during a 6-year exposure period. The results show that always low levels are independently associated with about a fourfold high risk of $\mathrm{Ml}$ in people with diabetes and pre-diabetes after adjusting for major confounding factors whereas participants who maintained high levels of eGFR throughout the exposure period had the lowest risk of incident MI. Moreover, participants kept moderate levels of eGFR which had like those in participants from high levels of eGFR to moderate levels at last exposure period, the risk of MI was much higher in eGFR decreasing group. Notably, compared with the lowest risk group, participants who started with moderate levels of eGFR that increased substantially also had an increased risk of incident MI, although their eGFR levels were within the normal range.

There is growing awareness that eGFR progression is an important risk factor for end stage renal disease (ESKD), cardiovascular disease and all-cause mortality. Previous studies have illustrated large variation in 
diabetes-dependent decline in eGFR with or without albuminuria[23]. In Earlier years, there is already reported that a rapid decline in kidney function is an independent risk of all-cause mortality[24]. Accordingly, recent studies have shown the relationship between eGFR slopes which estimated by multiple measurements of eGFR and the subsequent risk of ESKD[25], cardiovascular disease[12] and allcause mortality[24, 26, 27]. In 2019, Megumi Oshima et al. reported that eGFR slope as a prognostic factor for identifying individuals at high risk of cardiovascular disease and all-cause mortality with type 2 diabetes participants[10].

Similarly, our study identified individuals eGFR trajectories, and found a rapid decrease in eGFR levels may had a higher risk of MI. However, we also found participants with increasing normal eGFR levels can restore MI risk.

In this study, we assessed the associations between distinct trajectories of eGFR and the risk of MI with diabetes or pre-diabetes. The group-based trajectory modeling is a powerful statistical approach to describe the trajectories of eGFR over years. This approach could estimate the average, variability, and the direction of variability simultaneously in 1 model and allow us to investigate the population heterogeneity in longitudinal changes in eGFR levels, which may provide additional information. For example, participants with similar cumulative average of eGFR levels in a long period, a rapid decrease in eGFR levels may had a higher risk of MI than keep stable level. Moreover, participants with increasing eGFR levels even reach normal also had a risk of MI. We think these findings are essential because they would iditified different trajectories, encompass strategies reducing eGFR to prevent MI and provide critical implications for intervention for MI.

Mechanisms potentially underpinning the association between eGFR and the incidence of MI may be that eGFR reflects an impaired kidney function which has risk factors in common with cardiovascular disease. Furth more, various mechanisms have been suggested to explain the reason. Decrease in eGFR may indirectly influence the risk factors of cardiovascular, such as lower levels of blood pressure and lipids[28]. There are also other possible factors include activation of the RAAS, endothelial dysfunction, inflammation and oxidative stress[29, 30]. According these factors may progression of cardiovascular disease. In addition, worsening kidney function may cause decreased appetite and overall frailty, and indirectly result in decreased physical function[27].

The strengths of our study include the prospective design, large cohort, a long follow-up period, repeated measurements of eGFR levels, and use of eGFR changes to estimate the risk of MI. Our study also has several limitations. First, the study is an observational study design. Although we carefully adjusted for potential risks factors, the possibility of residual confounding remained. Second, this study lacks information about glycated hemoglobin and 2-h glucose due to the high cost for our large study samples, which is an important index for diagnosis of diabetes and prediabetes. Third, the disease duration and records of medications were not included in this study, which may influence the results. Finally, the population in the Kailuan cohort study are most of male coal miners. The unbalanced distribution of gender may have different demographic characteristics and risk factors; it is unknown whether our 
findings could be generalized to other ethnic groups; and further studies are warranted to verify our findings.

\section{Conclusions}

We found that distinct trajectories of eGFR were associated with MI risk in diabetic or pre-diabetic population. Our findings indicate that change in eGFR levels might play some extend reversible role in the early stage of the development of $\mathrm{Ml}$ and highlight the importance of monitoring longitudinal changes in eGFR levels in the primary prevention of MI. With the development of medicine, therapies targeted at eGFR remission for MI need further investigation.

\section{Abbreviations}

Estimated glomerular filtration rate, eGFR

Myocardial infarction, MI

Chronic kidney disease, CKD

African American Study of Kidney Disease and Hypertension, AASK

Hazard ratio, HR

Confidence intervals, Cls

Serum creatinine, Scr

Chronic Kidney Disease Epidemiological Collaboration, CKD-EPI

Electrocardiogram, ECG

Fasting blood glucose, FBG

Blood pressure, BP

Body mass index, BMI

Systolic blood pressure, SBP

Diastolic blood pressure, DBP

Total cholesterol, TC

Triglycerides, TG

Low-density lipoprotein cholesterol, LDL-C 
High-density lipoprotein cholesterol, HDL-C

End stage renal disease, ESKD

\section{Declarations}

- Ethics approval and consent to participate

The study followed the guidelines of the Helsinki Declaration, and was approved by the Ethics Committees of both the Kailuan General Hospital and Beijing Tiantan Hospital.

- Consent for publication

Not applicable

- Availability of data and material

The datasets used and/or analysed during the current study are available from the corresponding author on reasonable request.

- Competing interests

The authors declare that they have no competing interests.

- Funding

This study was funded by National Natural Science Foundation of China (31672375).

- Authors' contributions

$\mathrm{YZ}$ and $\mathrm{AW}$ wrote the manuscript. $\mathrm{YZ}$ researched data. SC and $\mathrm{XT}$ researched data and contributed to discussion. SW reviewed and edited the manuscript. YH contributed to the discussion and reviewed/edited the manuscript. All authors read and approved the final manuscript.

- Acknowledgements

We thank all the staff and participants of the Kailuan study for their invaluable contributions.

\section{References}

1. Zhang L, Wang F, Wang L, Wang W, Liu B, Liu J, Chen M, He Q, Liao Y, Yu X, et al. Prevalence of chronic kidney disease in China: a cross-sectional survey. Lancet. 2012;379(9818):815-22.

2. Pugliese G, Penno G, Natali A, Barutta F, Di Paolo S, Reboldi G, Gesualdo L, De Nicola L: Diabetic kidney disease: New clinical and therapeutic issues. Joint position statement of the Italian Diabetes Society and the Italian Society of Nephrology on "The natural history of diabetic kidney disease and 
treatment of hyperglycemia in patients with type 2 diabetes and impaired renal function". Nutrition, metabolism, and cardiovascular diseases: NMCD 2019, 29(11):1127-1150.

3. Sarnak MJ, Levey AS, Schoolwerth AC, Coresh J, Culleton B, Hamm LL, McCullough PA, Kasiske BL, Kelepouris E, Klag MJ, et al: Kidney disease as a risk factor for development of cardiovascular disease: a statement from the American Heart Association Councils on Kidney in Cardiovascular Disease, High Blood Pressure Research, Clinical Cardiology, and Epidemiology and Prevention. Hypertension (Dallas, Tex. 1979) 2003, 42(5):1050-1065.

4. Kannel WB, Stampfer MJ, Castelli WP, Verter J. The prognostic significance of proteinuria: the Framingham study. American heart journal. 1984;108(5):1347-52.

5. Chang SH, Tsai CT, Yen AM, Lei MH, Chen HH, Tseng CD. Proteinuria and Reduced Estimated Glomerular Filtration Rate Independently Predict Risk for Acute Myocardial Infarction: Findings from a Population-Based Study in Keelung, Taiwan. Acta Cardiologica Sinica. 2015;31(2):106-12.

6. Vinnakota S, Scott CG, Rodeheffer RJ, Chen HH: Estimated Glomerular Filtration Rate, Activation of Cardiac Biomarkers and Long-Term Cardiovascular Outcomes: A Population-Based Cohort. Mayo Clinic proceedings 2019, 94(11):2189-2198.

7. Puddu PE, Bilancio G, Terradura Vagnarelli O, Lombardi C, Mancini M, Zanchetti A, Menotti A. Serum uric acid and eGFR_CKDEPI differently predict long-term cardiovascular events and all causes of deaths in a residential cohort. Int J Cardiol. 2014;171(3):361-7.

8. Li Z, Wang A, Cai J, Gao X, Zhou Y, Luo Y, Wu S, Zhao X. Impact of proteinuria and glomerular filtration rate on risk of ischaemic and intracerebral hemorrhagic stroke: a result from the Kailuan study. European journal of neurology. 2015;22(2):355-60.

9. Matsushita K, van der Velde M, Astor BC, Woodward M, Levey AS, de Jong PE, Coresh J, Gansevoort RT. Association of estimated glomerular filtration rate and albuminuria with all-cause and cardiovascular mortality in general population cohorts: a collaborative meta-analysis. Lancet. 2010;375(9731):2073-81.

10. Oshima M, Jun M, Ohkuma T, Toyama T, Wada T, Cooper ME, Hadjadj S, Hamet P, Harrap S, Mancia $\mathrm{G}$, et al. The relationship between eGFR slope and subsequent risk of vascular outcomes and allcause mortality in type 2 diabetes: the ADVANCE-ON study. Diabetologia. 2019;62(11):1988-97.

11. El Dayem SM, Battah AA, El Bohy Ael M, El Shehaby A, El Ghaffar EA. Relationship of plasma level of chemerin and vaspin to early atherosclerotic changes and cardiac autonomic neuropathy in adolescent type 1 diabetic patients. Journal of pediatric endocrinology metabolism: JPEM. 2015;28(3-4):265-73.

12. Turin TC, Jun M, James MT, Tonelli M, Coresh J, Manns BJ, Hemmelgarn BR. Magnitude of rate of change in kidney function and future risk of cardiovascular events. Int J Cardiol. 2016;202:657-65.

13. Li L, Astor BC, Lewis J, Hu B, Appel LJ, Lipkowitz MS, Toto RD, Wang X, Wright JT Jr, Greene TH. Longitudinal progression trajectory of GFR among patients with CKD. American journal of kidney diseases: the official journal of the National Kidney Foundation. 2012;59(4):504-12. 
14. Davis TM, Chubb SA, Davis WA. The relationship between estimated glomerular filtration rate trajectory and all-cause mortality in type 2 diabetes: the Fremantle Diabetes Study. European journal of endocrinology. 2016;175(4):273-85.

15. Jiang G, Luk AOY, Tam CHT, Xie F, Carstensen B, Lau ESH, Lim CKP, Lee HM, Ng ACW, Ng MCY, et al. Progression of diabetic kidney disease and trajectory of kidney function decline in Chinese patients with Type 2 diabetes. Kidney international. 2019;95(1):178-87.

16. Echouffo-Tcheugui JB, Narayan KM, Weisman D, Golden SH, Jaar BG. Association between prediabetes and risk of chronic kidney disease: a systematic review and meta-analysis. Diabetic medicine: a journal of the British Diabetic Association. 2016;33(12):1615-24.

17. Wang A, Wu J, Zhou Y, Guo X, Luo Y, Wu S, Zhao X. Measures of adiposity and risk of stroke in China: a result from the Kailuan study. PloS one. 2013;8(4):e61665.

18. Levey AS, Stevens LA, Schmid CH, Zhang YL, Castro AF 3rd, Feldman HI, Kusek JW, Eggers P, Van Lente $F$, Greene T, et al. A new equation to estimate glomerular filtration rate. Ann Intern Med. 2009;150(9):604-12.

19. Fourth universal definition of myocardial infarction (2018). Revista espanola de cardiologia (English ed) 2019, 72(1):72.

20. Nagin DS, Odgers CL. Group-based trajectory modeling in clinical research. Ann Rev Clin Psychol. 2010;6:109-38.

21. Jones BL, Nagin DS, Roeder K: A SAS Procedure Based on Mixture Models for Estimating Developmental Trajectories. Sociological Methods \& Research 2001.

22. Nieto FJ, Coresh J. Adjusting survival curves for confounders: a review and a new method. Am J Epidemiol. 1996;143(10):1059-68.

23. Vistisen D, Andersen GS, Hulman A, Persson F, Rossing P, Jorgensen ME. Progressive Decline in Estimated Glomerular Filtration Rate in Patients With Diabetes After Moderate Loss in Kidney
Function-Even Without Albuminuria. 2019, 42(10):1886-1894.

24. Turin TC, Coresh J, Tonelli M, Stevens PE, de Jong PE, Farmer CK, Matsushita K, Hemmelgarn BR. Change in the estimated glomerular filtration rate over time and risk of all-cause mortality. Kidney international. 2013;83(4):684-91.

25. Kovesdy CP, Coresh J, Ballew SH, Woodward M, Levin A, Naimark DM, Nally J, Rothenbacher D, Stengel B, Iseki K, et al. Past Decline Versus Current eGFR and Subsequent ESRD Risk. Journal of the American Society of Nephrology: JASN. 2016;27(8):2447-55.

26. Al-Aly Z, Zeringue A, Fu J, Rauchman MI, McDonald JR, El-Achkar TM, Balasubramanian S, Nurutdinova D, Xian H, Stroupe K, et al. Rate of kidney function decline associates with mortality. Journal of the American Society of Nephrology: JASN. 2010;21(11):1961-9.

27. Naimark DM, Grams ME, Matsushita K, Black C, Drion I, Fox CS, Inker LA, Ishani A, Jee SH, Kitamura $A$, et al. Past Decline Versus Current eGFR and Subsequent Mortality Risk. Journal of the American Society of Nephrology: JASN. 2016;27(8):2456-66. 
28. Matsushita K, Selvin E, Bash LD, Franceschini N, Astor BC, Coresh J. Change in estimated GFR associates with coronary heart disease and mortality. Journal of the American Society of Nephrology: JASN. 2009;20(12):2617-24.

29. Schiffrin EL, Lipman ML, Mann JF. Chronic kidney disease: effects on the cardiovascular system. Circulation. 2007;116(1):85-97.

30. Navarro-Gonzalez JF, Mora-Fernandez C, Muros de Fuentes M, Garcia-Perez J. Inflammatory molecules and pathways in the pathogenesis of diabetic nephropathy. Nature reviews Nephrology. $2011 ; 7(6): 327-40$.

\section{Tables}

Table 1. Characteristics of the study participants at baseline by eGFR trajectory.

\begin{tabular}{|c|c|c|c|c|c|c|c|}
\hline Characteristic & Total & Low-stable & $\begin{array}{l}\text { Moderate- } \\
\text { stable }\end{array}$ & $\begin{array}{l}\text { Moderate- } \\
\text { increasing }\end{array}$ & $\begin{array}{l}\text { High- } \\
\text { decreasing }\end{array}$ & High-stable & $P$ value \\
\hline $\mathrm{N}$ & 24723 & 1943 & 8306 & 7831 & 2833 & 3810 & \\
\hline Age, years, median (IQR) & $\begin{array}{l}52.1(45.3- \\
58.7)\end{array}$ & $\begin{array}{l}59.3(52.0- \\
67.9)\end{array}$ & $\begin{array}{l}55.7(49.4- \\
64.1)\end{array}$ & $52.1(46.8-57.1)$ & $\begin{array}{l}51.8(45.3- \\
56.4)\end{array}$ & $\begin{array}{l}43.4(36.4- \\
49.4)\end{array}$ & $<0.01$ \\
\hline Male sex, n (\%) & 20655(83.5) & 1367(70.4) & $6945(83.6)$ & $6482(82.8)$ & $2488(87.8)$ & $3373(88.5)$ & $<0.01$ \\
\hline Current smoker, n (\%) & 8958(37.0) & $448(23.4)$ & $2546(31.4)$ & $3097(40.3)$ & $971(35.5)$ & $1896(50.0)$ & $<0.01$ \\
\hline Current alcohol, n (\%) & $10021(41.4)$ & $434(22.7)$ & $2667(32.9)$ & $3586(46.7)$ & $1111(40.7)$ & $2223(58.8)$ & $<0.01$ \\
\hline \multicolumn{8}{|l|}{ Physical activity, n (\%) } \\
\hline Inactive & $2440(10.2)$ & $98(5.2)$ & $585(7.3)$ & 744(9.8) & 403(14.8) & $610(16.2)$ & $<0.01$ \\
\hline Moderately active & 17433(72.7) & $1365(72.3)$ & $5686(70.9)$ & $5556(73.4)$ & $2028(74.4)$ & $2798(74.2)$ & \\
\hline Very active & $4102(17.1)$ & $425(22.5)$ & $1747(21.8)$ & $1271(16.8)$ & $294(10.8)$ & $365(9.7)$ & \\
\hline Dyslipidemia, n (\%) & $10693(43.2)$ & $970(50.0)$ & $3680(44.3)$ & $3483(44.5)$ & $1132(40.0)$ & $1428(37.5)$ & $<0.01$ \\
\hline Hypertension, n (\%) & $12959(52.4)$ & $1380(71.0)$ & $5210(62.7)$ & $3707(47.3)$ & $1367(48.2)$ & $1295(34.0)$ & $<0.01$ \\
\hline Diabetes mellitus, n (\%) & 7331(29.6) & $849(43.7)$ & $2659(32.0)$ & $2314(29.6)$ & $674(23.8)$ & $835(22.0)$ & $<0.01$ \\
\hline Proteinuria, n (\%) & $1199(4.8)$ & $199(10.2)$ & $426(5.1)$ & $284(3.6)$ & $143(5.0)$ & $147(3.9)$ & $<0.01$ \\
\hline FBG, mmol/L, median (IQR) & $6.2(5.8-7.1)$ & $6.4(5.9-8.2)$ & $6.2(5.8-7.2)$ & $6.1(5.8-7.1)$ & $6.1(5.8-6.8)$ & $6.1(5.8-6.6)$ & $<0.01$ \\
\hline LDL, mmol/L, median (IQR) & $2.5(2.0-3.0)$ & $2.5(2.1-3.0)$ & $2.5(2.1-3.0)$ & $2.4(1.9-3.0)$ & $2.5(2.1-2.9)$ & $2.4(2.1-2.8)$ & $<0.01$ \\
\hline $\mathrm{HDL}, \mathrm{mmol} / \mathrm{L}$, median (IQR) & $1.5(1.3-1.7)$ & $1.6(1.4-1.9)$ & $1.5(1.3-1.8)$ & $1.4(1.2-1.7)$ & $1.4(1.3-1.7)$ & $1.5(1.3-1.7)$ & $<0.01$ \\
\hline $\mathrm{TG}, \mathrm{mmol} / \mathrm{L}$, median (IQR) & $1.5(1.0-2.3)$ & $1.7(1.2-2.8)$ & $1.5(1.1-2.3)$ & $1.5(1.0-2.2)$ & $1.4(1.0-2.1)$ & $1.4(0.9-2.2)$ & $<0.01$ \\
\hline $\mathrm{TC}, \mathrm{mmol} / \mathrm{L}$, median (IQR) & $5.1(4.4-5.8)$ & $5.0(4.3-5.7)$ & $5.1(4.4-5.8)$ & $5.2(4.5-5.9)$ & $5.1(4.5-5.8)$ & $5.0(4.3-5.7)$ & $<0.01$ \\
\hline SBP, mmHg, median (IQR) & $\begin{array}{l}130.0(120.0- \\
149.3)\end{array}$ & $\begin{array}{l}140.0(130.0- \\
160.0)\end{array}$ & $\begin{array}{l}140.0(122.7- \\
151.7)\end{array}$ & $\begin{array}{l}130.0(120.0- \\
143.3)\end{array}$ & $\begin{array}{l}130.0(120.0- \\
141.3)\end{array}$ & $\begin{array}{l}121.7(113.3- \\
135.0)\end{array}$ & $<0.01$ \\
\hline DBP, mmHg, median (IQR) & $\begin{array}{l}82.0(80.0- \\
90.0)\end{array}$ & $\begin{array}{l}90.0(80.0- \\
98.0)\end{array}$ & $\begin{array}{l}87.0(80.0- \\
95.0)\end{array}$ & 81.3(79.3-90.0) & $\begin{array}{l}82.0(80.0- \\
90.0)\end{array}$ & $\begin{array}{l}80.0(76.0- \\
90.0)\end{array}$ & $<0.01$ \\
\hline BMI, $\mathrm{kg} / \mathrm{m}^{2}$, median (IQR) & $\begin{array}{l}25.7(23.5- \\
27.9)\end{array}$ & $\begin{array}{l}26.1(24.0- \\
28.4)\end{array}$ & $\begin{array}{l}25.8(23.7- \\
28.0)\end{array}$ & $25.7(23.6-28.0)$ & $\begin{array}{l}25.2(23.1- \\
27.4)\end{array}$ & $\begin{array}{l}25.2(22.8- \\
27.5)\end{array}$ & $<0.01$ \\
\hline $\begin{array}{l}\text { eGFR } \\
\left(\mathrm{ml} / \mathrm{min} / 1.73 \mathrm{~m}^{2}\right), \quad \text { median } \\
(\mathrm{IQR})\end{array}$ & $\begin{array}{l}81.7(68.0- \\
97.3)\end{array}$ & $\begin{array}{l}54.6(46.8- \\
60.3)\end{array}$ & $\begin{array}{l}70.0(62.3- \\
78.2)\end{array}$ & $83.0(74.5-90.9)$ & $\begin{array}{l}102.8(97.8- \\
108.6)\end{array}$ & $\begin{array}{l}107.0(100.2- \\
114.5)\end{array}$ & $<0.01$ \\
\hline $\begin{array}{l}\text { Uric acid, umol/L, median } \\
\text { (IQR) }\end{array}$ & $\begin{array}{l}282.3(231.7- \\
341.0)\end{array}$ & $\begin{array}{l}283.0(235.0- \\
351.0)\end{array}$ & $\begin{array}{l}285.0(233.0- \\
334.0)\end{array}$ & $\begin{array}{l}291.0(238.9- \\
351.0)\end{array}$ & $\begin{array}{l}266.0(216.0- \\
318.5)\end{array}$ & $\begin{array}{l}274.0(226.0- \\
330.0)\end{array}$ & $<0.01$ \\
\hline $\begin{array}{l}\text { Antihypertensive medication, } \\
\mathrm{n}(\%)\end{array}$ & $3501(14.2)$ & $442(22.8)$ & 1323(15.9) & $1145(14.6)$ & $292(10.3)$ & $299(7.8)$ & $<0.01$ \\
\hline $\begin{array}{l}\text { Antidiabetic medication, } \mathrm{n} \\
(\%)\end{array}$ & $1809(7.3)$ & $273(14.0)$ & $638(7.7)$ & $645(8.2)$ & $102(3.6)$ & $151(4.0)$ & $<0.01$ \\
\hline $\begin{array}{l}\text { Lipid-lowering medication, } \mathrm{n} \\
(\%)\end{array}$ & $316(1.3)$ & $43(2.2)$ & $106(1.3)$ & 103(1.3) & $31(1.1)$ & $33(0.9)$ & $<0.01$ \\
\hline ACEI medication, $\mathrm{n}(\%)$ & $206(0.8)$ & $21(1.1)$ & $70(0.9)$ & $87(1.2)$ & $13(0.5)$ & $15(0.4)$ & $<0.01$ \\
\hline
\end{tabular}

Abbreviations: ACEI, angiotensin converting enzyme inhibitors; BMI, body mass index; DBP, diastolic blood pressure; eGFR, estimated glomerular filtration rate; FBG, fasting blood glucose; HDL, high-density lipoprotein cholesterol; LDL, low-density lipoprotein cholesterol; SBP, systolic blood pressure; TC, total cholesterol; TG, triglycerides. 
Table 2. Hazard ratios of trajectories of eGFR for incident myocardial infarction.

\begin{tabular}{|c|c|c|c|c|c|}
\hline \multirow[t]{2}{*}{ Variable } & \multicolumn{5}{|c|}{ eGFR trajectories } \\
\hline & Low-stable & Moderate-stable & Moderate-increasing & High-decreasing & High-stable \\
\hline \multicolumn{6}{|l|}{ Myocardial infarction } \\
\hline Case, $\mathrm{n}(\%)$ & $40(2.1)$ & $94(1.1)$ & $62(0.8)$ & $31(1.1)$ & $8(0.2)$ \\
\hline $\begin{array}{l}\text { Incidence rate, } \\
\text { per 1000-person, y }(95 \% \mathrm{CI})\end{array}$ & $4.71(3.45-6.42)$ & $2.47(2.02-3.03)$ & $1.70(1.33-2.18)$ & $2.36(1.66-3.36)$ & $0.45(0.22-0.89)$ \\
\hline Model 1 & $10.5(4.91-22.42)$ & $5.52(2.68-11.37)$ & $3.80(1.82-7.94)$ & $5.28(2.43-11.49)$ & Reference \\
\hline Model 2 & $5.97(2.69-13.26)$ & $3.42(1.62-7.20)$ & $2.78(1.32-5.87)$ & $3.82(1.74-8.38)$ & Reference \\
\hline Model 3 & $4.54(2.03-10.12)$ & $2.82(1.34-5.95)$ & $2.55(1.21-5.39)$ & $3.43(1.56-7.54)$ & Reference \\
\hline \multicolumn{6}{|l|}{ Sensitivity analysis } \\
\hline Sensitivity analysis 1 & $5.36(2.04-14.12)$ & $2.88(1.18-7.03)$ & $2.86(1.20-6.86)$ & $3.50(1.41-8.69)$ & Reference \\
\hline Sensitivity analysis 2 & $4.32(1.73-10.79)$ & $2.61(1.14-6.02)$ & $2.54(1.12-5.74)$ & $3.10(1.32-7.28)$ & Reference \\
\hline
\end{tabular}

Abbreviations: eGFR, estimated glomerular filtration rate.

Model 1: Unadjusted.

Model 2: Adjusted for age and gender in 2006.

Model 3: Model 2+; additional adjusted for current smoker, current alcohol, physical activity, proteinuria, ACEI medication and body mass index, fasting blood glucose, systolic blood pressure and total cholesterol in 2006.

Sensitivity analysis 1: Adjusted for age, gender, current smoker, current alcohol, physical activity, proteinuria, ACEI medication and body mass index, fasting blood glucose, systolic blood pressure and total cholesterol in 2012.

Sensitivity analysis 2: Adjusted for age, gender, current smoker, current alcohol, physical activity, proteinuria and ACEI medication in 2012 average body mass index, average fasting blood glucose, average systolic blood pressure average total cholesterol during the exposure period.

Table 3. Hazard ratios of trajectories of eGFR for incident myocardial infarction with stratification by baseline characteristics.

\begin{tabular}{|c|c|c|c|c|c|c|}
\hline \multirow[t]{2}{*}{ Variable } & \multicolumn{5}{|c|}{ eGFR trajectories } & \multirow{2}{*}{$\begin{array}{l}P \text { for } \\
\text { interaction }\end{array}$} \\
\hline & Low-stable & Moderate-stable & Moderate-increasing & High-decreasing & High-stable & \\
\hline \multicolumn{7}{|c|}{ (6) } \\
\hline$<55$ years & $4.59(1.66-12.72)$ & $2.21(0.94-5.18)$ & $2.52(1.10-5.75)$ & $3.79(1.57-9.14)$ & Reference & 0.47 \\
\hline$\geq 55$ years & $4.45(0.60-33.19)$ & $3.03(0.42-22.02)$ & $2.17(0.30-16.01)$ & $2.55(0.33-19.61)$ & Reference & \\
\hline \multicolumn{7}{|l|}{ Gender } \\
\hline Men & $4.88(2.06-11.54)$ & $3.06(1.38-6.79)$ & $2.84(1.28-6.29)$ & $3.70(1.60-8.54)$ & Reference & 0.87 \\
\hline Women & $1.99(0.22-18.09)$ & $1.13(1.13-9.87)$ & $0.75(0.08-6.98)$ & $1.56(0.14-17.76)$ & Reference & \\
\hline \multicolumn{7}{|l|}{ Hypertension } \\
\hline No & $4.72(1.37-16.24)$ & $1.99(0.69-5.75)$ & $2.04(0.75-5.55)$ & $4.22(1.49-11.95)$ & Reference & 0.24 \\
\hline Yes & $5.42(1.61-18.27)$ & $3.80(1.18-12.27)$ & $3.44(1.06-11.18)$ & $3.50(1.01-12.10)$ & Reference & \\
\hline \multicolumn{7}{|l|}{ Diabetes } \\
\hline No & $5.05(1.77-14.39)$ & $3.29(1.28-8.48)$ & 2.93(1.14-7.53) & $3.83(1.42-10.28)$ & Reference & 0.98 \\
\hline Yes & $3.75(1.06-13.29)$ & $2.24(0.67-7.54)$ & $2.06(0.61-6.96)$ & $2.94(0.80-10.84)$ & Reference & \\
\hline \multicolumn{7}{|l|}{ Proteinuria } \\
\hline No & $4.51(1.80-11.31)$ & $3.26(1.39-7.64)$ & $3.24(1.39-7.58)$ & $3.83(1.56-9.39)$ & Reference & 0.17 \\
\hline Yes & $3.29(0.61-17.87)$ & $1.16(0.22-5.99)$ & $0.22(0.02-2.48)$ & $2.17(0.40-11.71)$ & Reference & \\
\hline
\end{tabular}

Abbreviations: eGFR, estimated glomerular filtration rate.

Adjusted for age, gender, current smoker, current alcohol, physical activity, proteinuria, ACEI medication and body mass index, fasting blood glucose, systolic blood pressure and total cholesterol in 2006.

\section{Figures}




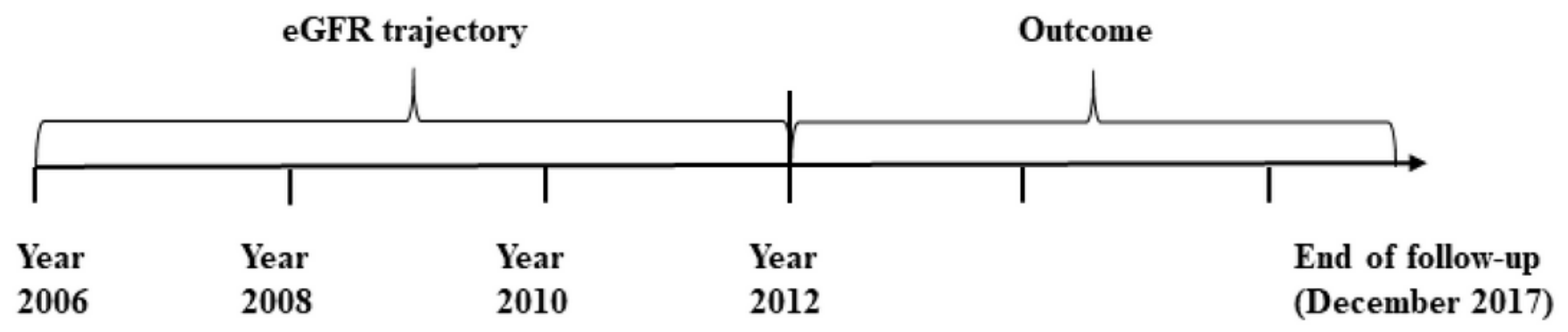

Figure 1

Flowchart of the study cohort. Abbreviations: eGFR, estimated glomerular filtration rate.

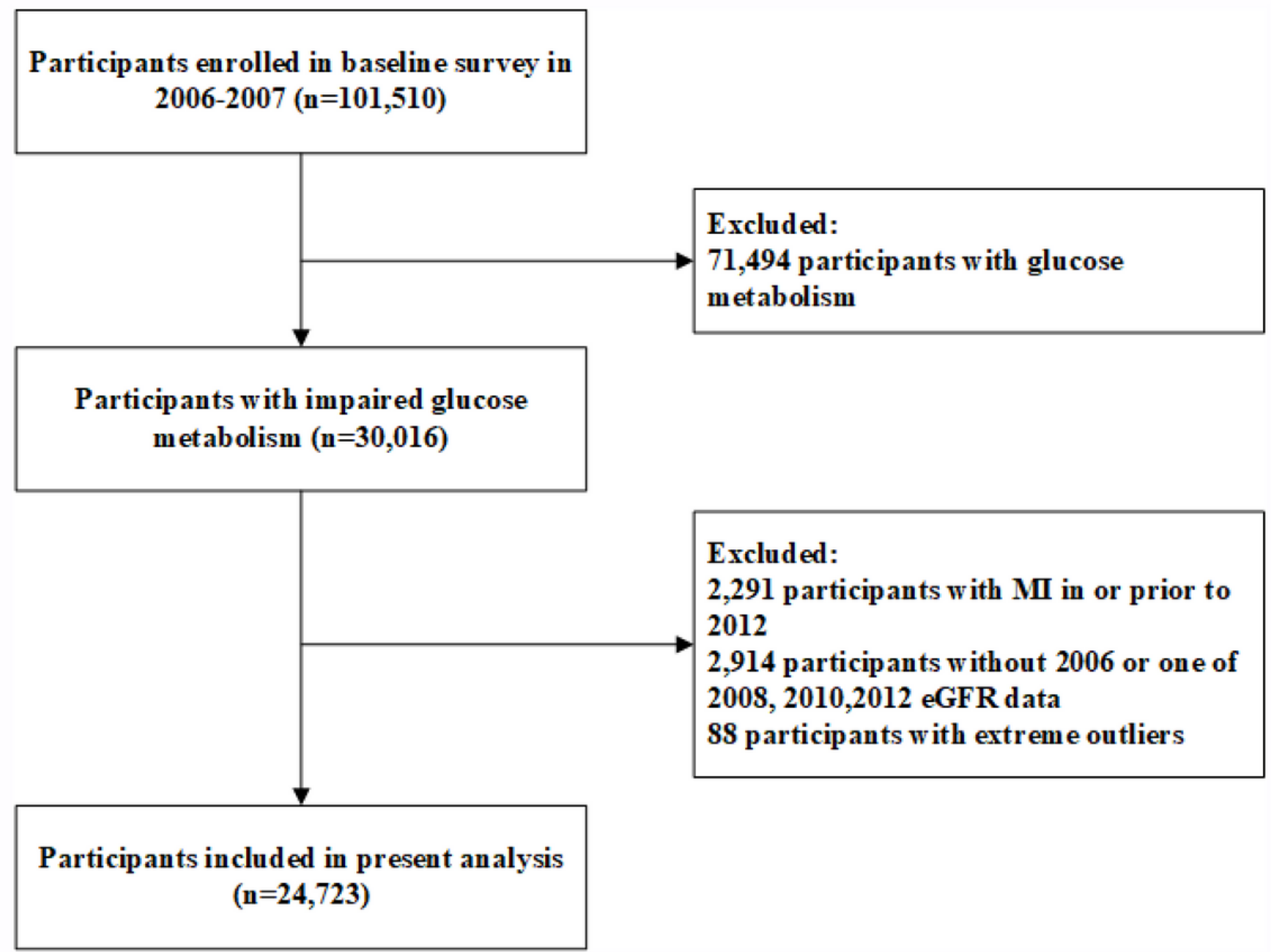

Figure 2 
Time line of the study. Abbreviations: eGFR, estimated glomerular filtration rate; MI, Myocardial infarction.

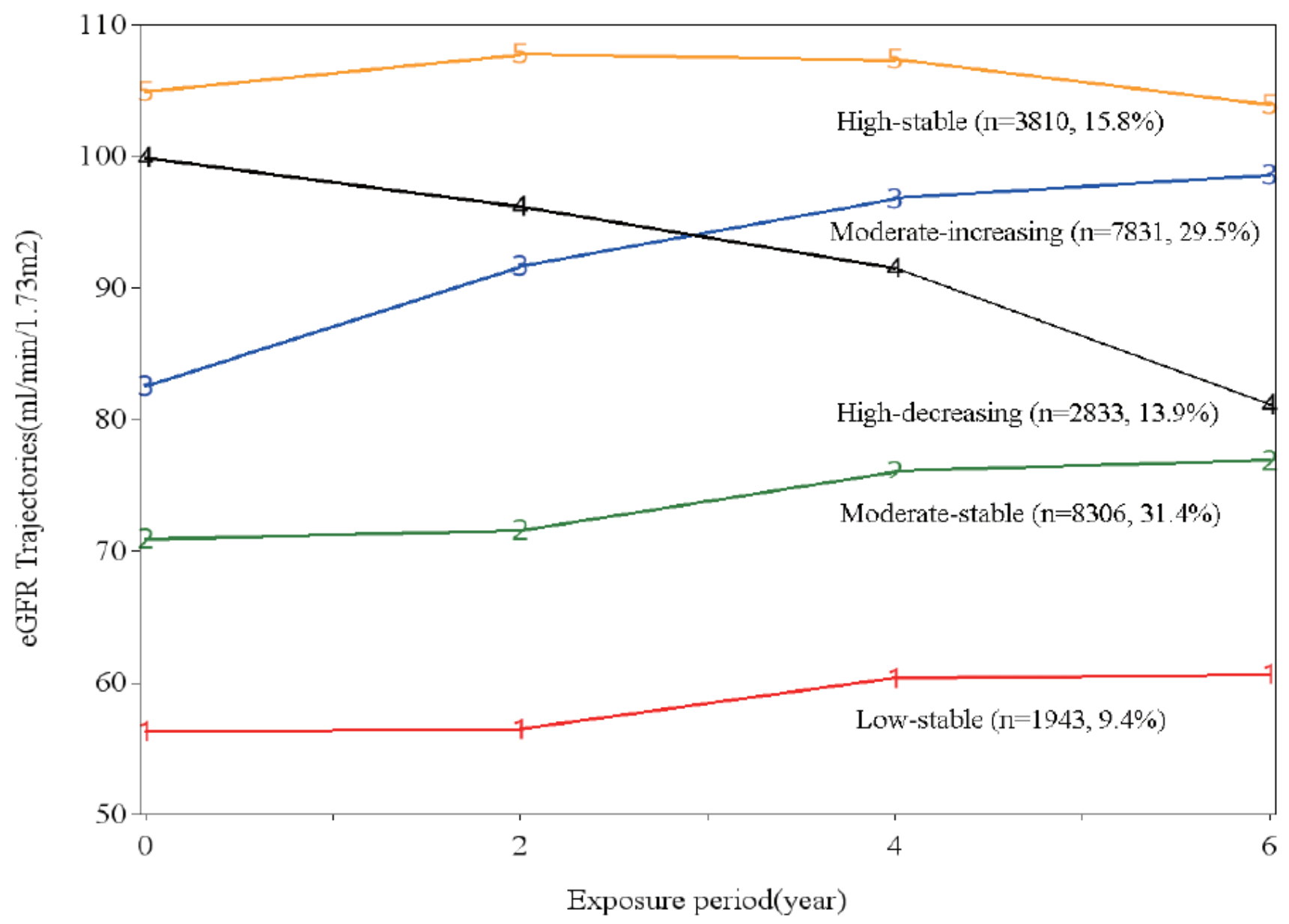

Figure 3

Trajectories of eGFR over 6 years. Abbreviations: eGFR, estimated glomerular filtration rate. 


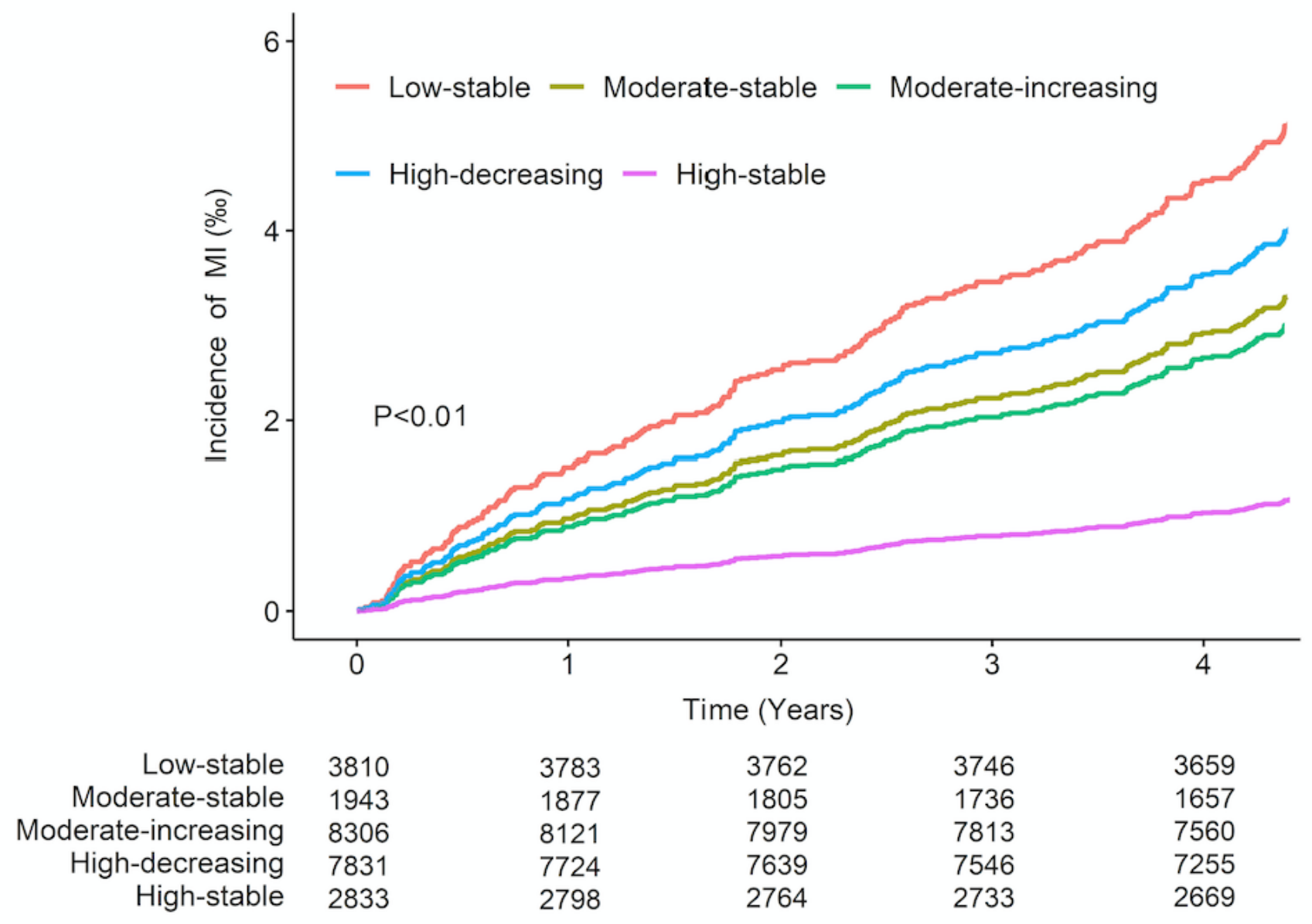

Figure 4

Survival curves of trajectories of eGFR and myocardial infarction. Abbreviations: eGFR, estimated glomerular filtration rate. Adjusted for age, gender, current smoker, current alcohol, physical activity, proteinuria, ACEI medication and body mass index, fasting blood glucose, systolic blood pressure and total cholesterol in 2006. 